\title{
Bridging reduziert nicht das Schlaganfallrisiko, erhöht aber das Blutungsrisiko
}

Fragestellung: Ist die Unterbrechung einer oralen Antikoagulation mit Vitamin-K-Antagonisten (VKA) bei Patienten, die sich einem elektiven operativen Eingriff unterziehen müssen, genauso wirksam zur Reduktion des Schlaganfallrisikos wie das klassische Bridging mit niedermolekularem Heparin?

Hintergrund: Patienten mit Vorhofflimmern haben ein hohes Schlaganfallrisiko, dass durch eine orale Antikoagulation, beispielsweise mit VKA, signifikant reduziert werden kann. Bei geplanten operativen Eingriffen ist es bisher üblich, dass ein sogenanntes Bridging durchgeführt wird. Dabei wird der VKA etwa fünf Tage vor dem operativen Eingriff abgesetzt und fünf bis zehn Tage nach der Operation wieder angesetzt. In der Zwischenzeit werden die Patienten mit niedermolekularem Heparin (NMH) behandelt. Das NMH wird nur am Tag des Eingriffs pausiert. Auf diese Weise soll das Risiko von perioperativen Schlaganfällen minimiert werden. Interessanterweise gab es bis jetzt noch keine randomisierte Studie, die dieses Behandlungskonzept validiert oder ein Bridging mit einem einfachen Unterbrechen der Antikoagulation verglichen hätte.

Patienten und Methodik: Die BRIDGE-Studie war eine randomisierte, doppelblinde, placebokontrollierte Studie, in die 1.884 Patienten eingeschlos-

Douketis JD, Spyropoulos AC, Kaatz $S$ et al; BRIDGE Investigators. Perioperative bridging anticoagulation in patients with atrial fibrillation. N Engl J Med 2015; 373: $823-33$ sen wurden, die sich einem elektiven operativen Eingriff unterziehen mussten. Die Patienten wurden in zwei Therapiegruppen randomisiert. Bei der einen Gruppe wurde der VKA Warfarin drei Tage vor dem Eingriff abgesetzt und fünf bis zehn Tage nach dem Eingriff wieder angesetzt. Die Zwischenzeit wurde mit Ausnahme des Tages der Operation mit 100 Einheiten Dalteparin pro kg Körpergewicht überbrückt. Die Vergleichsgruppe erhielt statt des niedermolekularen Heparins subkutane Placeboinjektionen. Die Patienten wurden dann 30 Tage lang nachbeobachtet. Der primäre Endpunkt waren Schlaganfälle, transiente ischämische Attacken, systemische Embolien und schwerwiegende Blutungskomplikationen. In die Studie wurden 1.884 Patienten eingeschlossen, von denen 913 mit Placebo und 891 mit Verum behandelt wurden. Die Patienten waren im Mittel 72 Jahre alt, $73 \%$ waren Männer. Ein Drittel der Patienten nahm zusätzlich zu Warfarin Acetylsalicylsäure ein.

Arterielle Thromboembolien traten bei vier Patienten $(0,4 \%)$ in der Gruppe ohne Bridging und bei drei Patienten (0,3\%) in der Gruppe mit Bridging auf. Schwerwiegende Blutungskomplikationen gab es bei zwölf Patienten (1,3\%), in der Gruppe ohne Bridging und bei 29 Patienten (3,2\%) in der Gruppe mit Bridging. Dieser Unterschied war mit einem p-Wert von 0,005 signifikant. Es ergaben sich keine signifikanten Unterschiede für Tod, Herzinfarkt und tiefe Beinvenenthrombosen sowie Lungenembolien.

Schlussfolgerungen: Bei Patienten, die wegen Vorhofflimmern mit VKA antikoaguliert sind, erhöht eine einfache Unterbrechung der Antikoagulation aufgrund einer elektiven Operation oder eines invasiven diagnostischen Eingriffs das Risiko eines Schlaganfalls nicht, verglichen mit dem üblichen Bridging mit niedermolekularem Heparin. Eine einfache Therapieunterbrechung reduziert aber signifikant das Risiko schwerwiegender Blutungen.

\section{- Kommentar von Hans-Christoph Diener, Essen}

\section{Auch etablierte Therapien müssen kritisch hinterfragt werden}

Diese sehr gut durchgeführte Studie ist ein weiteres Beispiel dafür, dass die Vorgehensweise in der klinischen Medizin immer wieder kritisch hinterfragt werden sollte und lang geübte Therapien, die auch in Leitlinien empfohlen werden, einer kritischen Überprüfung im Rahmen von randomisierten Studien bedürfen. Bisher bestand fast kein Zweifel, dass Patienten die wegen Vorhofflimmern antikoaguliert werden, bei einem operativen Eingriff oder einer invasiven Prozedur zwischenzeitlich mit niedermolekularem Heparin vor einem Schlaganfall geschützt werden müssen. Die BRIDGE-Studie zeigt nun eindeutig, dass dieses Konzept falsch ist, da das Schlaganfallrisiko bei einer einfachen Unterbrechung der Antikoagulation nicht erhöht ist, das Bridging allerdings zu einer signifikanten Steigerung von Blutungskomplikationen führt. Dieses Ergebnis ist auch für die Neurologie relevant, da beispielsweise bei Liquorpunktionen unter Antikoagulation mit VKA kein Bridging notwendig ist.

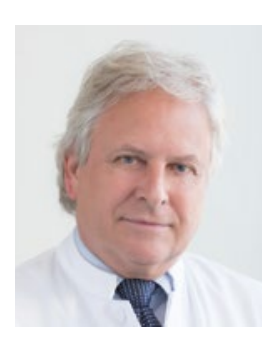

Prof. Dr. med. Hans-Christoph Diener, Essen

Direktor der Klinik für Neurologie, Universitätsklinikum Essen E-Mail:h.diener@uni-essen.de 